\title{
Optical gain observation on silicon nanocrystals embedded in silicon nitride under femtosecond pumping
}

B. M. Monroy, O. Crégut, M. Gallart, B. Hönerlage, and P. Gilliot

Citation: Appl. Phys. Lett. 98, 261108 (2011); doi: 10.1063/1.3607276

View online: https://doi.org/10.1063/1.3607276

View Table of Contents: http://aip.scitation.org/toc/apl/98/26

Published by the American Institute of Physics

\section{Articles you may be interested in}

Photoluminescence responses of Si nanocrystal to differing pumping conditions

Journal of Applied Physics 110, 013502 (2011); 10.1063/1.3601350

Silicon quantum wire array fabrication by electrochemical and chemical dissolution of wafers

Applied Physics Letters 57, 1046 (1990); 10.1063/1.103561

Enhancing optical gains in Si nanocrystals via hydrogenation and cerium ion doping Journal of Applied Physics 116, 043512 (2014); 10.1063/1.4891506

Stimulated emission and optical gain in $\mathrm{ZnO}$ epilayers grown by plasma-assisted molecular-beam epitaxy with buffers

Applied Physics Letters 78, 1469 (2001); 10.1063/1.1355665

DIRECT DETERMINATION OF OPTICAL GAIN IN SEMICONDUCTOR CRYSTALS

Applied Physics Letters 18, 475 (1971); 10.1063/1.1653501

Effect of hydrogen on the photoluminescence of Si nanocrystals embedded in a $\mathrm{SiO}_{2}$ matrix

Applied Physics Letters 78, 1225 (2001); 10.1063/1.1338492

\section{PHYSICS TODAY}

MANAGER'S GUIDE

WHITEPAPERS

\section{READ NOW}

PRESENTED BY

Multiphysics Simulation 


\title{
Optical gain observation on silicon nanocrystals embedded in silicon nitride under femtosecond pumping
}

\author{
B. M. Monroy, ${ }^{1, a)}$ O. Crégut, ${ }^{2}$ M. Gallart, ${ }^{2}$ B. Hönerlage, ${ }^{2}$ and P. Gilliot ${ }^{2}$ \\ ${ }^{1}$ Instituto de Investigaciones en Materiales, Universidad Nacional Autónoma de México, AP 70-360, \\ Coyoacán, CP 04510, Distrito Federal, México \\ ${ }^{2}$ Institut de Physique et Chimie des Matériaux de Strasbourg, Unité Mixte CNRS-ULP (UMR 7504), \\ 23 rue du Loess, BP 43, F-670 34 Strasbourg Cedex 2, France
}

(Received 22 March 2011; accepted 11 June 2011; published online 1 July 2011)

\begin{abstract}
We report the observation of positive optical gain in silicon nanocrystals (Si-nc) embedded in silicon nitride measured by the variable stripe length technique. We evidence the onset of stimulated emission and report gain coefficients up to $52 \mathrm{~cm}^{-1}$ at the highest excitation power $(6.5$ $\mathrm{W} / \mathrm{cm}^{2}$ ). Photoluminescence dynamics presents two distinct recombination lifetimes in the nanosecond and the microsecond ranges. This was interpreted in terms of fast carrier trapping in nitrogen-induced localized states in the Si-nc surface and subsequent slow radiative recombination, suggesting that carrier trapping in radiative surface states plays a crucial role in the optical gain mechanism of Si-nc. (C) 2011 American Institute of Physics. [doi:10.1063/1.3607276]
\end{abstract}

Obtaining an efficient light source from a silicon-based material would enable opto-electronics integration at low cost. Since the first report of optical gain in silicon nanocrystals (Si-nc), ${ }^{1}$ various groups have independently confirmed this observation in $\mathrm{Si}$-nc embedded in silicon oxide $\left(\mathrm{SiO}_{2}\right)$ obtained by different methods ${ }^{1-5}$ or in $\mathrm{Si}-\mathrm{nc} / \mathrm{SiO}_{2}$ superlattices. $^{2,6}$ Optical gain coefficients from 10 to $100 \mathrm{~cm}^{-1}$ have been measured mainly by the variable-stripe-length method $\left(\mathrm{VSL}^{7}\right.$ or by pump-probe experiments. ${ }^{1}$ A four-level phenomenological model has been proposed which takes into account the strong competition between Auger recombination and stimulated emission in these systems. ${ }^{3}$ Oxygenrelated states introduced at the interface $\mathrm{Si}-\mathrm{nc} / \mathrm{SiO}_{2}$ matrix may play a crucial role in the observation of stimulated emission. Nevertheless, it is not clear whether the optical gain is intrinsic to $\mathrm{Si}$-nc or related to the oxide passivation, and the material conditions for reaching positive optical gain still remain unclear. Si-nc embedded in silicon nitride $\left(\mathrm{SiN}_{\mathrm{x}}\right)$ present intense photoluminescence (PL) in the nanosecond range, ${ }^{8}$ tunable in the visible spectrum. ${ }^{9}$ Electrical current injection is expected to be easier in $\mathrm{SiN}_{\mathrm{x}}$, leading to more efficient electroluminescence. However, no observation of optical amplification in $\mathrm{Si}-\mathrm{nc} / \mathrm{SiN}_{\mathrm{x}}$ has been reported to date, despite some initial efforts. ${ }^{10}$ In this letter, we report the observation of positive optical gain in the $\mathrm{Si}-\mathrm{nc} / \mathrm{SiN}_{\mathrm{x}}$ system. We evidence the onset of stimulated emission at $550 \mathrm{~nm}$ and report gain coefficients up to $52 \mathrm{~cm}^{-1}$ at the highest excitation power. The observation of optical gain is related to the PL decay mechanism of the samples, which is similar to $\mathrm{Si}$-nc/SiO 2 systems.

The $\mathrm{SiN}_{\mathrm{x}}$ film was deposited using a plasma-enhanced chemical vapor deposition system. The deposition conditions are described elsewhere. ${ }^{11}$ The film thickness was $830 \mathrm{~nm}$ and the index of refraction measured at $633 \mathrm{~nm}$ was 1.95 . The excess concentration of silicon in the film was $17.7 \%$ and the estimated concentration of Si-nc in the matrix was $\sim 1.2 \times 10^{18} \mathrm{~cm}^{-3}$. Si-nc have a mean diameter of $3.2 \mathrm{~nm}$

${ }^{\text {a)} E l e c t r o n i c ~ m a i l: ~ m a r e l @ i i m . u n a m . m x . ~}$ and a size distribution between 1 and $4 \mathrm{~nm} .{ }^{11}$ There was 5.6 at. $\%$ of $\mathrm{Cl}$ incorporated in the film, improving the surface passivation of Si-nc. ${ }^{12,13}$ Optical gain was evaluated using the VSL configuration. ${ }^{7}$ The excitation pulses $\left(\lambda_{\text {exc }}=382\right.$ $\mathrm{nm}$, duration $100 \mathrm{fs}$, repetition rate $200 \mathrm{kHz}$ ) were delivered by a frequency-doubled Ti-sapphire oscillator. The beam was focused into a rectangular stripe of $\sim 60 \mu \mathrm{m} \times 1.5 \mathrm{~mm}$ on the sample surface. PL emission from the sample facet was collected with a microscope objective focused into a spectrometer. Pumping powers of $6.5,4.6$, and $2.8 \mathrm{~W} / \mathrm{cm}^{2}$ were used. The pump powers required to achieve gain with fs pulses can be relatively low due to reduction of thermal phenomena and high rates of carrier injection. ${ }^{4}$ In order to eliminate possible parasitic effects in the VSL measurement, ${ }^{14,16}$ we used the shifting-excitation-spot (SES) technique. SES measurements were used for the correct optical alignment of the detection optics and to eliminate other measurement artifacts. ${ }^{5}$ Integrated PL decay dynamics was measured using a photomultiplier tube with resolution of 2 $\mathrm{ns}$ and a streak camera with resolution better than $50 \mathrm{ps}$, taking into account the jitter between the pulses. All measurements were done at room temperature and corrected for the spectral response of the systems.

Ideally, the SES signal $\left(I_{S E S}\right)$ is given by $I_{S E S}=I_{0}$ $(\lambda) \cdot \exp \left[-\alpha_{\text {Tot }}(\lambda) \cdot x\right]$, where $I_{0}(\lambda)$ is the spontaneous emission, $x$ is the excitation spot position from the sample's edge, and $\alpha_{\text {Tot }}(\lambda)=\alpha(\lambda)+K$ represents the optical losses due to absorption and scattering. The difference between the VSL and the integrated SES curves can provide information about the net gain coefficient $G(\lambda)$ following the model of Eq. (1). ${ }^{5}$ If no stimulated emission is present in the sample, the SES signal integrated over the stripe length $(l)$ should give exactly the same result as the VSL method $\left(I_{V S L}\right)$. If stimulated emission is present, the difference of the VSL and integrated SES curves increases exponentially with $l$. For increasing excitation intensities, the population of the excited state increases with respect to that of the ground state and population inversion can be reached. Then, stimulated emission occurs: material intrinsic absorption $\alpha$ turns into gain $g_{\text {mat }}$ when its sign is 
changed. Since the losses $K$ due to scattering still remain, the net gain coefficient that is measured experimentally and that is shown in Eq. (1) is given by $G=g_{\text {mat }}-K$. In this case, the total optical losses in the film, estimated from the SES data, were $\alpha_{\text {Tot }}=20 \pm 2 \mathrm{~cm}^{-1}$ for all emission wavelengths,

$$
\begin{aligned}
I_{V L S}(l, \lambda) & -\int_{0}^{l} I_{S E S}(x, \lambda) d x=I_{0}(\lambda) \\
& \times\left(\frac{e^{G(\lambda) \cdot l}-1}{G(\lambda)}-\frac{1-e^{-\alpha_{T o t}(\lambda) \cdot l}}{\alpha_{T o t}(\lambda)}\right) .
\end{aligned}
$$

VSL and integrated SES curves are compared at $550 \mathrm{~nm}$ (Figure 1(a)) and $700 \mathrm{~nm}$ (Figure 1(c)). For $550 \mathrm{~nm}$, the data coincide at $2.8 \mathrm{~W} / \mathrm{cm}^{2}$, clearly indicating the absence of net gain. At $6.5 \mathrm{~W} / \mathrm{cm}^{2}$, the initial parts of the VSL and integrated SES curves are identical, but after a threshold stripe length of $250 \mu \mathrm{m}$, the integrated SES lies evidently under the VSL curve, indicating the stimulated emission onset. For 4.6 $\mathrm{W} / \mathrm{cm}^{2}$, the stimulated emission occurrence cannot be unambiguously determined. Figure 1(b) shows the difference between VSL and integrated SES curves. The gain coefficient $G$ was obtained by fitting the data using Eq. (1). To avoid artifacts due to diffraction on the movable slits, the data up to the threshold length were not considered in the fit. Optical losses of $-5 \mathrm{~cm}^{-1}$ are obtained for $2.8 \mathrm{~W} / \mathrm{cm}^{2}$, while positive optical gain of $+18 \mathrm{~cm}^{-1}$ is observed for $6.5 \mathrm{~W} /$ $\mathrm{cm}^{2}$. For $700 \mathrm{~nm}$, there is an exponential increase of the VSL curves (Figure 1(c)), while the integrated SES curves increase sublinearly. A positive difference between both curves is clear after $150 \mu \mathrm{m}$ and it increases with pumping power (Figure 1(d)), which constitutes clear evidence for stimulated emission observation. ${ }^{5}$ Saturation is observed in all cases $\sim 500 \mu \mathrm{m}$. $G$ was estimated by fitting the difference from the threshold length until the saturation length, because saturation effects are not included in Eq. (1). ${ }^{14,16}$ The optical gain coefficient reaches $52 \mathrm{~cm}^{-1}$ at $6.5 \mathrm{~W} / \mathrm{cm}^{2}$.

Figure 2(a) features the PL spectrum detected at the sample facet, where no waveguiding effects such as narrowing of the PL edge emission with respect to the normal geometry were observed (as in Ref. 15). Only the interference
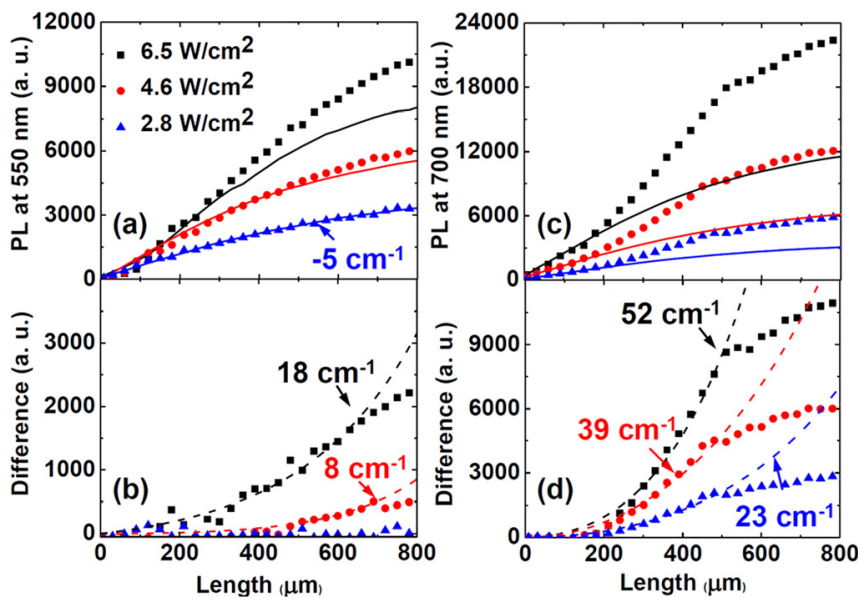

FIG. 1. (Color online) VSL (symbols) and integrated SES curves (lines) at detection wavelengths of (a) $550 \mathrm{~nm}$ and (c) $700 \mathrm{~nm}$. Difference between VSL and integrated SES curves and fit for gain at (b) $550 \mathrm{~nm}$ and (d) $700 \mathrm{~nm}$. due to the multiple reflections at the film-substrate and airfilm interfaces can be appreciated. No spectral narrowing with increasing excitation intensity was observed in our case. This effect could be screened by the inhomogeneous broadening of the PL spectrum due to the wide size distribution of Si-nc in the film. Gain spectra are presented in Figure 2(b) in the region from 525 to $750 \mathrm{~nm}$. In this case, no blueshift from the gain spectrum with respect to the PL emission is appreciated, as observed in Ref. 3, but there is a slight spectral narrowing from $180 \mathrm{~nm}$ to $160 \mathrm{~nm}$. This is consistent with the fact that the mean Si-nc size in our sample is 3.2 $\mathrm{nm}$, and we have a broad size distribution. On the other hand, the gain spectra in Figure 2(b) increase with excitation power as expected since population inversion strongly depends on the pumping conditions. ${ }^{14,16}$

Integrated PL dynamics was measured in the microsecond (Figure 3(a)) and nanosecond (Figure 3(b)) regimes. There are two distinct PL decay times in the film: a "slow" radiative lifetime of $1.08 \pm 0.5 \mu$ s that has a stretched exponential behavior $(\beta=0.66 \pm 0.03)$ and a "fast" radiative lifetime of $0.5 \pm 0.2 \mathrm{~ns}$. Shorter picoseconds time decays can also be present but they are not observed here because of the time resolution of the experiment. The "slow" recombination dynamics is similar to the one observed in $\mathrm{Si}-\mathrm{nc} / \mathrm{SiO}_{2}$ systems, ${ }^{3,5}$ where the variation of the atomic structure of oxidized Si-nc could influence the local competition between radiative and nonradiative rates and, thus, be responsible for the stretched exponential behavior of the PL decay. ${ }^{17}$ The observation of a $\mu$ s-stretched exponential decay is an important difference between the PL dynamics that we observe in our $\mathrm{Si}$-nc and other $\mathrm{SiN}_{\mathrm{x}}$ films evaluated for optical gain, ${ }^{10}$ while the fast nanosecond decay is typical of Si-nc embedded in $\mathrm{SiN}_{\mathrm{x}} \cdot{ }^{8}$ Theoretical calculations show that radiative lifetimes in Si-nc are significantly influenced by bridging-nitrogen configurations at the nanocrystals surface. Depending on the

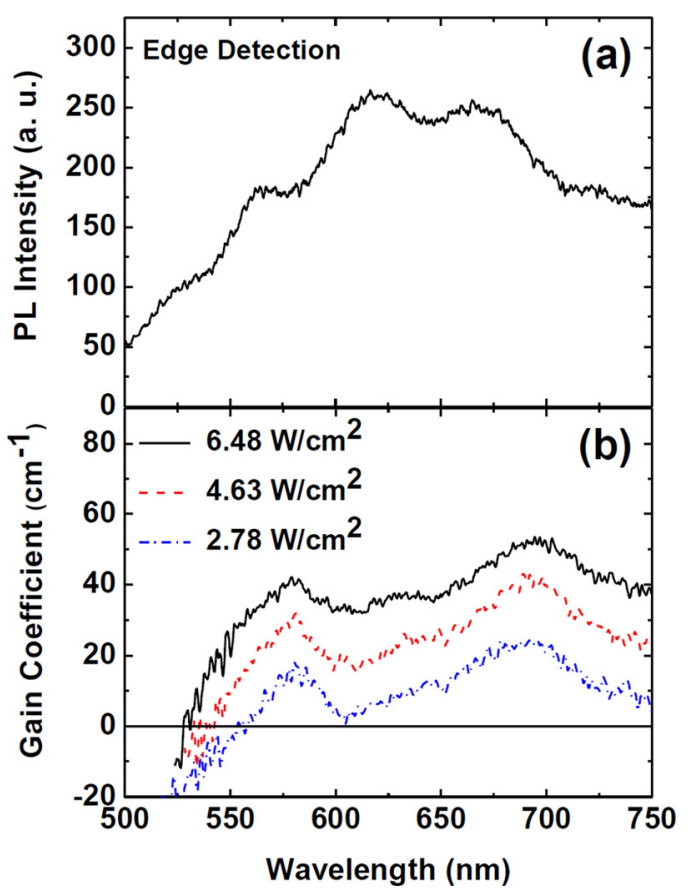

FIG. 2. (Color online) (a) PL emission detected at the sample facet. (b) Gain spectra at different excitation powers. 

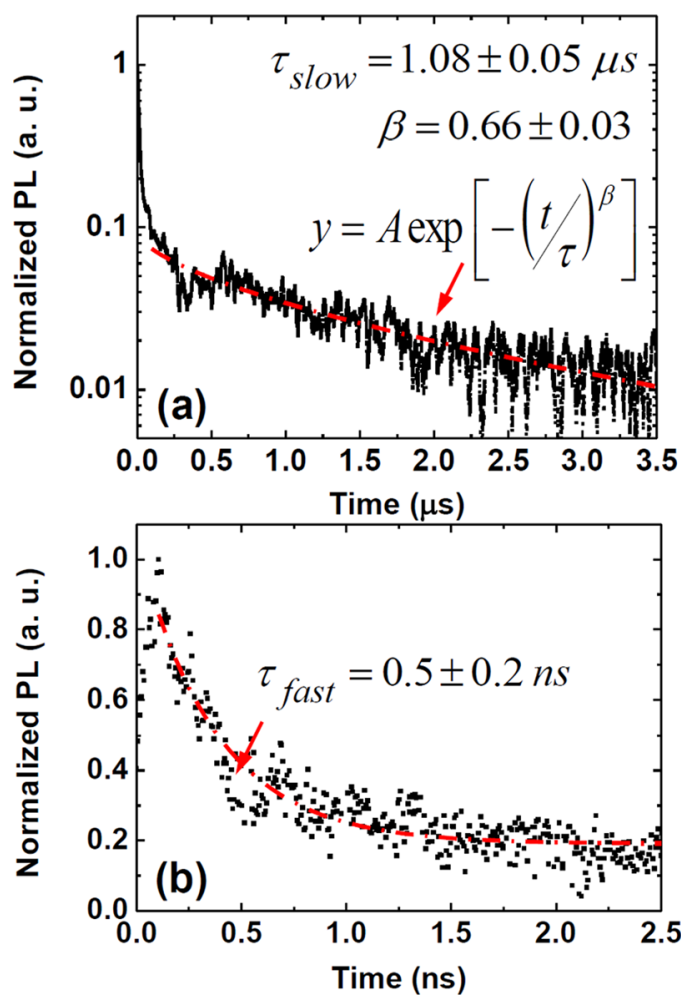

FIG. 3. (Color online) PL decay dynamics at (a) the microsecond range and (b) the nanosecond range.

nitrogen surface passivation, localized states introduced within the optical gap of Si-nc cause nitrogen trap states responsible for light emission. ${ }^{18}$ Therefore, we interpret the light emission mechanism in our $\mathrm{SiN}_{\mathrm{x}}$ film as follows: the fast nanosecond PL decay component is associated with the nonradiative exciton trapping on the radiative nitrogen states present at the Si-nc surface while the longer microsecond decay results from the recombination, both radiative and nonradiative, of the trapped excitons. This interpretation implies that the trapped carriers are spatially separated, which leads to a reduced electron-hole wavefunctions overlap and a suppressed Auger mechanism. ${ }^{19}$ This effect depends on the type of surface passivation, since different nitrogen-bonding configurations at the Si-nc surface lead to different energy states within the confined optical band gap. ${ }^{8,20}$ The PL dynamics in our samples resembles the one observed in Si-nc embedded in $\mathrm{SiO}_{2}$, but at shorter decay times. Thus, the optical gain mechanism could be similar. In particular, it seems that carrier trapping in radiative surface states induced by the Si-nc passivation plays a crucial role.

In conclusion, we observed optical amplification in the VSL configuration from Si-nc embedded in $\mathrm{SiN}_{\mathrm{x}}$, with a maximum gain coefficient of $52 \mathrm{~cm}^{-1}$ at $700 \mathrm{~nm}$ for the highest excitation power. The gain spectra increase with the excitation power reflecting the dependence of population inversion with the pumping conditions. The PL dynamics was interpreted in terms of nanosecond carrier trapping in nitrogen-induced localized states in the Si-nc surface and subsequent microsecond radiative recombination. This suggests that carrier trapping in radiative surface states induced by the Si-nc passivation (either by oxygen or nitrogen) plays a crucial role in the gain mechanism of Si-nc.

The authors acknowledge the partial financial support of this work from ICyTDF Project PICCO10-73 and DGAPAIACOD \#I1100411. Special thanks are due to J. Camacho, O. Jiménez, M. T. Vázquez, and C. González for technical and information services.

${ }^{1}$ L. Pavesi, L. Dal Negro, C. Mazzoleni, G. Franzo, and F. Priolo, Nature (London) 408, 440 (2000).

${ }^{2}$ P. M. Fauchet, J. Ruan, H. Chen, L. Pavesi, L. Dal Negro, M. Cazzaneli, R. G. Elliman, N. Smith, M. Samoc, and B. Luther-Davies, Opt. Mater. 27, 745 (2005).

${ }^{3}$ L. Dal Negro, M. Cazzanelli, N. Daldosso, Z. Gaburro, L. Pavesi, F. Priolo, D. Pacifici, G. Franzò, and F. Iacona, Physica E 16, 297 (2003).

${ }^{4}$ K. Luterová, K. Dohnalová, V. Švrček, I. Pelant, J.-P. Likforman, O. Crégut, P. Gilliot, and B. Hönerlage, Appl. Phys. Lett. 84, 3280 (2004).

${ }^{5}$ K. Dohnalová, I. Pelant, K. Kůsová, P. Gilliot, M. Gallart, O. Crégut, J.-L. Rehspringer, B. Hönerlage, T. Ostatnický, and S. Bakardjeva, New J. Phys. 10, 063014 (2008).

${ }^{6}$ M. Cazzanelli, D. Navarro-Urriós, F. Riboli, N. Daldosso, L. Pavesi, J. Heitmann, L. X. Yi, R. Scholz, M. Zacharias, and U. Gösele, J. Appl. Phys. 96, 3164 (2004).

${ }^{7}$ K. L. Shaklee, R. E. Nahory, and R. F. Leheny, J. Lumin 7, 284 (1973).

${ }^{8}$ L. Dal Negro, J. H. Yi, L. C. Kimerling, S. Hamel, A. Williamson, and G. Galli, Appl. Phys. Lett. 88, 183103 (2006).

${ }^{9}$ T.-W. Kim, C.-H. Cho, B.-H. Kim, and S.-J. Park, Appl. Phys. Lett. 88, 123102 (2006).

${ }^{10}$ H. Chen, J. H. Shin, P. M. Fauchet, J.-Y. Sung, J.-H. Shin, and G. Y. Sung, Appl. Phys. Lett. 91, 173121 (2007).

${ }^{11}$ A. Benami, G. Santana, A. Ortiz, A. Ponce, D. Romeu, J. Aguilar-Hernández, G. Contreras-Puente, and J. C. Alonso, Nanotechnology 18, 155704 (2007).

${ }^{12}$ B. M. Monroy, G. Santana, A. Benami, A. Ortiz, J. C. Alonso, J. Fandiño, F. Cruz-Gandarilla, J. Aguilar-Hernández, G. Contreras-Puente, A. LópezSuárez, and A. Oliver, J. Nanosci. Nanotechnol. 9, 2902 (2009).

${ }^{13}$ G. Santana, B. M. Monroy, A. Ortiz, L. Huerta, J. C. Alonso, J. Fandiño, J. Aguilar-Hernández, E. Hoyos, F. Cruz-Gandarilla, and G. ContrerasPuente, Appl. Phys. Lett. 88, 041916 (2006).

${ }^{14}$ J. Valenta, I. Pelant, and J. Linnros, Appl. Phys. Lett. 81, 1396 (2002).

${ }^{15}$ J. Valenta, I. Pelant, K. Luterova, R. Tomasiunas, S. Cheylan, R. G. Elliman, J. Linnos, and B. Hönerlage, Appl. Phys. Lett. 82, 955 (2003).

${ }^{16}$ L. Dal Negro, P. Bettotti, M. Cazzanelli, D. Pacifici, and L. Pavesi, Opt. Commun. 229, 337 (2004).

${ }^{17}$ O. Guillois, N. Herlin-Boime, C. Reynaud, G. Ledoux, and F. Huisken, J. Appl. Phys. 95, 3677 (2004).

${ }^{18}$ L. Dal Negro, J. H. Yi, J. Michel, L. C. Kimerling, T.-W. F. Chang, V. Sukhovatkin, and E. H. Sargent, Appl. Phys. Lett. 88, 233109 (2006).

${ }^{19}$ K. Dohnalová, K. Kůsová, O. Cibulka, L. Ondič, and I. Pelant, Phys. Scr., T 141, 014011 (2010).

${ }^{20}$ A. Martínez, J. C. Alonso, L. E. Sansores, and R. Salcedo, J. Phys. Chem. C 114, 12427 (2010). 